\title{
Desarrollo del sector servicios y su papel en la consolidación del crecimiento económico mundial
}

Development of the service sector and its role in consolidating global economic growth

Andrés Fernando Jola Sánchez*

Recibido: 31/08/2012

Aprobado: 15/01/2013

Estudiante doctoral y associate instructor de Kelley School of Business, Indiana University, Estados Unidos [andres.jola@gmail.com]. 
Desarrollo del sector servicios y su papel en la consolidación del crecimiento económico mundial

ANDRÉS FERNANDO JOLA SÁNCHEZ

\section{Resumen}

Mediante un análisis econométrico de tipo panel se estudia la relación del comercio y la producción nacional de servicios frente a la productividad por trabajador y el crecimiento económico mundial. Se describen dos mecanismos en que los servicios, a través de la tecnología que incorporan a la estructura productiva de las economías nacionales, incrementan la productividad media por trabajador y promueven el crecimiento económico. Utilizando información para 124 países y un periodo que va desde 1970 a 2007, se halla evidencia significativa del papel de estos dos mecanismos: importación y producción doméstica de servicios en la consolidación de las productividades medias de las naciones. Además, se encuentra que los servicios de alto contenido en capital humano, en contraposición a las actividades económicas de los sectores primario y secundario, sustentan la mayor proporción del crecimiento de la productividad por trabajador en países de altos y medianos ingresos.

\section{Palabras clave:}

Modelos con datos panel, desarrollo económico, productividad agregada, industria de servicios.

\section{Abstract}

The relationship between domestic trade and service production, on one hand, and productivity per worker and world economic growth, on the other, is studied in this paper through a panel data econometric analysis. Two mechanisms are described where services, by incorporating technology in the productive structure of domestic economies, increase average productivity per worker and promote economic growth. Data corresponding to 124 countries, between 1970 and 2007 was analyzed. Significant evidence was found about the role of both imported services and domestic service production in consolidating the nations' average productivity. Likewise, services with a high content of human capital, as opposed to economic activities in the primary and secondary sectors, were found to underpin the largest share of productivity growth per worker in high and middle income countries.

\section{Key words:}

Panel data models, economic development, aggregate productivity, service industry.

Clasificación JEL: C23, 01, 04, 014 


\section{Introducción}

El crecimiento económico, su relación con la productividad por trabajador y el vínculo con el desarrollo del sector servicios, es el principal objeto de estudio en este documento.

La novedad de este trabajo es, por un lado, la vinculación conceptual que tiene la oferta de servicios, especialmente la de alto contenido en capital humano, vía producción nacional o importación, con el aumento de las posibilidades de producción nacional, y por el otro, el uso de una metodología de panel para mostrar que la relación anterior tiene sustento vía aumento de la productividad por trabajador.

Para el análisis propuesto se conformó una base de datos con información para 124 países y un periodo correspondiente a 1971-2007. Las variables más importantes relacionadas en el estudio son el valor agregado de los diferentes sectores de servicios catalogados como de alto capital humano o alta tecnología, clasificados así por el alto nivel de cualificación de su recurso humano y el uso intensivo de tecnologías de información y comunicación (TIC) en su generación (García y Menéndez, 1992), indicadores de la actividad comercial de servicios y las productividades medias por trabajador de cada país.

Los resultados encontrados en la formulación econométrica son fundamentales para el entendimiento del efecto del capital humano en la productividad media por trabajador, de la misma forma que el impacto de la transferencia internacional de conocimientos, a través del sector servicios, en la estructura productiva de los Estados con diferentes niveles de ingresos. En el campo internacional, los resultados aportan evidencia valiosa de la estructura de producción que países catalogados como "exitosos" o de altos ingresos tienen frente a la registrada por países en desarrollo y con niveles económicos bajos.

El documento se encuentra organizado de la siguiente forma: en la sección dos se hace una revisión de la literatura en materia de crecimiento económico y servicios, en el capítulo tres se expone el modelo conceptual para el análisis del sector terciario, el capítulo cuarto contiene la hipótesis de trabajo y los datos del estudio estadístico y, finalmente, en los acápites cinco y seis se exponen los resultados del modelo y las conclusiones significativas del estudio. 
Desarrollo del sector servicios y su papel en la consolidación del crecimiento económico mundial

ANDRÉS FERNANDO JOLA SÁNCHEZ

\section{Revisión bibliográfica}

Los servicios han sido una preocupación fundamental en el proceso de construcción de la literatura económica; desde autores de corte clásico, como Adam Smith y Marx, hasta otros como Samuelson, Kravis, Heston y Summers, han tomado interés en caracterizar las dinámicas circunscritas a las actividades de servicios. La diferenciación entre la producción de bienes y servicios ha sido materia de amplia discusión, con especial atención de las condiciones de productividad y de desarrollo tecnológico que imponen e impactan sus estructuras operativas (Bhagwati, 1984). A diferencia de los primeros trabajos en la materia que indicaban una relativa improductividad y un menor progreso tecnológico de los servicios con relación a la manufactura, en la literatura reciente, como se mostrará a continuación, han sido rebatidos ampliamente dichos hallazgos, mostrando además que los servicios propician una dinámica favorable en las condiciones de productividad y competitividad de las economías.

En términos generales, la relación entre el crecimiento económico y el sector servicios ha sido abordada en la literatura bajo tres orientaciones fundamentales. La primera, aquella en la que se sostiene que el crecimiento económico tiene sustento en el aumento de la productividad de la economía, y esta última en el desarrollo de los servicios; la segunda, la que halla evidencia de que, por medio del comercio internacional de servicios, es posible adoptar conocimientos de frontera e incrementar un mayor crecimiento económico del país doméstico; y, finalmente, aquella en la que los servicios son insumos esenciales de la cadena de valor de la economía y fundamentales para la producción eficiente, siendo estas líneas de trabajo los elementos de política y regulación, elementos indispensable que facilitan la difusión y apropiación de los servicios en la cadena de valor de las múltiples actividades económicas.

En la primera de las orientaciones, algunos de los autores que tratan esta temática son Banga \& Goldar (2004). Los resultados de su trabajo demuestran que, utilizando una función de producción de valor añadido o KLEMS (capital-labor-energía-materiales-servicios), los servicios hacen un gran aporte a la productividad del sector manufacturero en la India, especialmente en los años noventa. Dicha relación es explicada, según el autor, por la adopción de servicios de alta intensidad en capital humano en la manufactura, que afectaron positivamente la productividad y eficiencia de dicho sector. 
De forma similar, Niño (2005) plantea que sectores como los de telecomunicaciones, infraestructura, energía, transporte, financieros, empresariales y profesionales, son los promotores de la creciente productividad, tanto de servicios como de bienes. La tesis de Niño se basa en que sectores de servicios como los mencionados promueven una mayor generación de recursos humanos calificados, que a la larga aumentarán la capacidad productiva nacional. Otros autores han demostrado que existe un mayor crecimiento económico derivado del desarrollo de varios sectores de servicios. Por ejemplo, Waverman y Roller (2001) y Nicoletti y Scarpetta (2003) han hallado evidencia estadística para sustentar que una expansión en la oferta de servicios de telecomunicaciones, energía y transporte causa mayor tasa de crecimiento económico. De la misma forma, Demirgüc-Kunt, Levine et al. (1999) han demostrado dicha relación para los servicios financieros, de educación y salud.

En la segunda orientación descrita por la literatura, es decir, en el estudio entre el crecimiento económico y el comercio internacional de servicios, existen diferentes perspectivas. En 2005, la Organización para la Cooperación y el Desarrollo Económico (OECD, por sus siglas en inglés), por ejemplo, encontró evidencia de que el creciente auge de la actividad del comercio internacional ha permitido que diversas economías con sectores de servicios relativamente improductivos puedan "ponerse al día", mediante la contratación y vinculación de servicios extranjeros altamente productivos.

En dicha línea, Li, Greenaway y Hine (2005) muestran que servicios intensivos en conocimiento promueven el desarrollo de economías de escala en la producción y, por lo tanto, la importación de estos permite la adopción y transferencia de tecnología de frontera proveniente de países altamente productivos.

Por último, la tercera línea de investigación está concentrada en mostrar que es posible relacionar, no solo la actividad comercial de servicios con el crecimiento económico, como ya se refirió, sino también considerar que su regulación y promoción por medio de las políticas públicas son mecanismos para la efectiva adopción de los servicios en el aparato productivo. En esta línea se ha encontrado que existe una relación significativa y positiva entre el crecimiento económico y la apertura comercial de servicios como el de telecomunicaciones (Khoury \& Savvides, 2006), lo que se constata especialmente en países con ingresos medios y bajos. Se ha hallado sustento de una relación positiva entre el crecimiento económico de países con altos ingresos y el grado de apertura 
Desarrollo del sector servicios y su papel en la consolidación del crecimiento económico mundial

ANDRÉS FERNANDO JOLA SÁNCHEZ

comercial del sector financiero. De forma similar, algunos autores (Eschenbach \& Hoekman, 2005) han mostrado cómo algunas reformas directas e indirectas que han facilitado el uso y comercialización de los servicios, han estado relacionadas con una buena proporción del desarrollo económico registrado en dichas economías. Específicamente, Hoekman y Mattoo (2000) plantean que, dado el hecho de que los servicios son importantes insumos para la producción de bienes y otros servicios, las políticas que afectan su calidad, precio y disponibilidad son absolutamente relevantes para el desempeño y competitividad de la economía.

\section{Formulación del modelo de servicios}

El equilibrio económico es, en cualquier caso, la interacción entre oferta y demanda, en la cual, como resultado, es posible obtener un vector de precios y cantidades que permiten asignar eficientemente los recursos de una economía. La actividad de servicios se encuentra sujeta a las fuerzas del mercado y no es ajena a esta dinámica de equilibrio económico; sin embargo, gran parte de los modelos macroeconómicos no se refieren a este sector directamente. Así las cosas, en el presente documento se motiva la inclusión directa del sector de servicios a este análisis macro.

En primer lugar, los servicios se adoptan en este ámbito bajo el concepto de representar un conjunto de actividades económicas en donde el "hacer" y el conocimiento del individuo constituyen la fuente principal de valor agregado. Dichas actividades, conocidas en la literatura como operaciones de servicios, tienen propiedades en común, como por ejemplo, la inseparabilidad entre la producción y el consumo, la imposibilidad de acumular la producción, la intangibilidad y, además, la relación inequívoca y uno a uno con el cliente.

En este trabajo, los servicios se analizarán en el entorno del modelo neoclásico dinámico de equilibrio competitivo, de la mano de todos sus supuestos y formas predeterminadas. ${ }^{1}$

1 Es importante precisar que el modelo planteado puede llegar a ser limitado en términos del análisis de la convergencia entre los países. Dicha estructuración conceptual requiere los siguiente supuestos:

- Rendimientos constantes a escala en la función de producción (homogeneidad de grado 1).

- La productividad marginal del capital, mano de obra y de servicios son positivas y decrecientes.

- La productividad marginal del trabajo es creciente ante un aumento de capital o de servicios.

- Las unidades de consumo e inversión son equivalentes en ambos periodos. 


\subsection{Modelo de oferta y demanda agregada con servicios}

El equilibrio competitivo se halla sujeto a la interacción entre oferta y demanda agregada. En el modelo, dicha oferta y demanda es, por un lado, el resultado de la función de producción de la economía, y por el otro, de la demanda de bienes de consumo, inversión, gasto público y exportaciones netas. Al incluir los servicios, por el lado de la demanda, estos son consumidos por los hogares, objeto de inversión por las firmas y comercializados en los mercados internacionales. Por el lado de la oferta, estos son producidos y adquiridos como insumos de producción.

La oferta agregada permitirá incorporar una primera novedad; la tecnología de la economía (Z) es función de los servicios y un mayor nivel de estos permitirá avanzar en una nueva frontera tecnológica. Nótese que los servicios pueden ser uno de muchos otros componentes como la innovación, que afectan la tecnología de la economía, y que para propósitos de este documento se mantendrán constantes.

$$
Y^{O}=Z(s) * f(k, n, s)^{B S}
$$

donde la función de producción representativa (1) incluye los insumos, $k=$ capital, $n=$ fuerza de trabajo

y $s=$ servicios

\subsection{Cambios en la productividad por cambios en la actividad de servicios}

Es fundamental suponer, en primer lugar, una función de ganancias $G$ de una firma representativa en dos periodos de tiempo, con decisiones solo sobre $n$, es decir, sobre la mano de obra. En dicha función la firma descuenta sus ganancias $\pi$ del segundo periodo a una tasa de interés $r$ (2-3). Además, en el periodo inicial existe un nivel dado de servicios, capital y mano de obra, con un pago $F_{\mathrm{i}}$ a los factores (capital y servicios) en cada uno de los periodos.

$$
\begin{aligned}
& G=\pi_{1}+\frac{\pi_{2}}{1+r} \\
& G=Z\left(s_{1}\right) * f\left(k_{1}, n_{1}, s_{1}\right)^{B S}-w_{1} * n_{1}-F_{1}
\end{aligned}
$$


Desarrollo del sector servicios y su papel en la consolidación

del crecimiento económico mundial

ANDRÉS FERNANDO JOLA SÁNCHEZ

$$
+\frac{Z\left(s_{2}\right) * f\left(k_{2}, n_{2}, s_{2}\right)^{B S}}{1+r}-w_{2} * n_{2}-F_{2}
$$

El producto por trabajador se define como las ganancias de este en cada periodo, teniendo, por lo tanto, que la productividad marginal por trabajador (PMGL), al maximizarse, tiene la siguiente estructura:

$$
\begin{aligned}
P M G L_{\mathrm{i}} & =\frac{d G}{d n_{i}}=\frac{d Z}{d s_{i}} * \frac{d s_{\mathrm{i}}}{d n_{i}} * f\left(k_{i}, n_{i}, s_{i}\right)^{B S} \\
& +Z\left(s_{\mathrm{i}}\right) f^{\prime}\left(k_{\mathrm{i}}, n_{\mathrm{i}}, s_{\mathrm{i}}\right)^{B S}
\end{aligned}
$$

En este resultado se puede observar que la expresión (5) es mayor a cero y se cumple gracias el supuesto inicial de que la tecnología de la economía es mejorada directamente por el nivel de servicios, que el stock de servicios se incrementa vía importaciones o producción nacional (6), y que se cumplen los supuestos fundamentales sobre rendimientos decrecientes de la función de producción. Es importante notar que, aunque se utiliza el concepto de stock, análogo al de inventarios, el significado en este documento no es el de almacenamiento, sino el de disponibilidad o capacidad de prestación. Es así que un mayor stock de servicios implica una mayor disponibilidad de los diferentes servicios en las actividades productivas y de consumo de la economía.

$$
\begin{aligned}
& \frac{d Z}{d s_{i}} * \frac{d s_{1}}{d n_{i}} * f\left(k_{i}, n_{i}, s_{i}\right)^{B S}+Z\left(s_{i}\right) f^{\prime}\left(k_{i}, n_{i}, s_{i}\right)^{B S} \\
& Y^{O \text { servicios }}=M^{s}+Z(s) * f(k, n, s)^{\text {Servicios }}
\end{aligned}
$$

Si los servicios no tuviesen una relación directa con la tecnología, es decir, si se cumple la expresión (7), la PMGL disminuiría en un término igual a $k$ (8) para cada uno de los periodos, lo que generaría un pago al factor similar al que se hace al capital y a la mano de obra. Por lo tanto, el aumento de la frontera tecnológica causado por el desarrollo del sector terciario es un aporte explícito al crecimiento económico doméstico según el modelo planteado.

$$
\begin{gathered}
\frac{d Z}{d s_{1}}=0 \\
\frac{d Z}{d s_{i}} * \frac{d s_{i}}{d n_{i}} * f\left(k_{i}, n_{i}, s_{i}\right)^{B S}=k
\end{gathered}
$$




\subsection{Canales de transmisión del crecimiento económico por cuenta del aumento de la disponibilidad de servicios}

De acuerdo con el modelo descrito, los servicios permiten mayor crecimiento económico cuanto mayor sea la disponibilidad de ellos, lo cual es posible vía su importación o producción doméstica.

Para mostrar este hecho se puede ver que, ante un incremento en el stock de servicios, por el lado de la oferta agregada habrá un incremento de la producción final de la economía al existir mayor disponibilidad de uno de sus insumos intermedios (servicios), que a su vez transforma la frontera de producción a un nivel tecnológico de la economía (Z’).

Por el lado de la demanda agregada podrán existir un par de efectos que incrementen su nivel. En primer lugar, la mayor generación y disponibilidad de servicios retribuye el capital humano y la labor; por lo tanto, de forma inducida esto llevará a incrementar el nivel de consumo privado. Por otro lado, las firmas, al pretender maximizar su producción dado el cambio tecnológico propiciado por los servicios, buscarán incrementar su inversión, lo cual generará, en cualquier caso, un incremento de la demanda agregada.

Ambos efectos llevarán a un aumento tanto de la oferta como de la demanda agregada, que en equilibrio, generará un crecimiento del producto interno de la economía igual a $\left(Y_{3}-Y_{1}\right) / Y_{1}$, como se muestra en la figura 1.

\section{Figura1.}

Equilibrio tras el incremento de los servicios de la economía

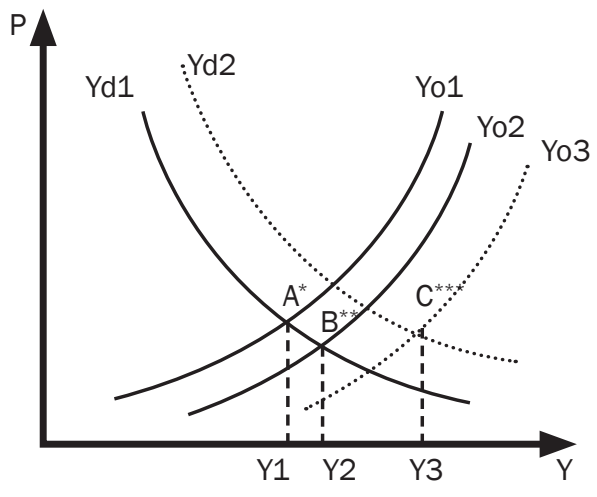

A* Es tado inicial de la economía $\mathrm{B}^{* *}$ Incremento de la oferta agregada por aumento del stock de servicios. $\mathrm{C} * * *$ Equilibrio con el incremento de la demanda y la oferta agregada por cuenta del cambio tecnológico, Yo: curva de oferta, Yd: curva de demanda. Fuente: elaboración propia 
Desarrollo del sector servicios y su papel en la consolidación del crecimiento económico mundial

ANDRÉS FERNANDO JOLA SÁNCHEZ

\subsection{Señales de crecimiento económico y actividad de servicios}

Se han planteado varias propiedades de los servicios que tienen repercusiones directas en el modelo descrito en este documento. Sin embargo, existe un elemento que no se ha tenido en cuenta por ahora, y es el de la exportación de servicios.

En el contexto en el cual se discuten los servicios, las importaciones desempeñan un papel fundamental al permitir la entrada de tecnología (conocimientos) a la producción nacional; ahora, la pregunta fundamental será: ¿qué tipo de señal o efecto tiene el ser un país exportador de servicios?

En este documento se plantea que la decisión de exportar servicios tendrá dependencia de alguna forma con estas tres variables:

1. La tasa de cambio.

2. Los términos de intercambio.

3. La viabilidad técnica de la prestación del servicio (incluidas las barreras legales y comerciales).

Bajo el supuesto de unos precios internacionales dados, la exportación será en últimas posible si existe una relación entre costos de producción y calidad adecuada, que es viable de alguna manera relacionar con las ventajas competitivas de la industria doméstica de servicios. De la mano de algunos modelos de comercio internacional, como el de Heckscher-Ohlin, es posible mostrar que el país que tenga una alta intensidad de conocimiento y tecnología tendrá una ventaja competitiva para exportar diversos tipos de servicios con alto contenido en dichos factores de producción. Por lo tanto, esto querría decir que países exportadores (de servicios) tienen industrias de servicios altamente productivas y de amplia disponibilidad.

\section{Modelo econométrico de panel}

De acuerdo con la hipótesis central descrita en la sección 3, un incremento de la disponibilidad de servicios (producción o importación) tendrá efecto positivo en la producti- 
vidad debido al crecimiento tecnológico que afectará finalmente la totalidad de bienes y servicios producidos en una economía. De esta forma, el objetivo del modelo será contrastar el efecto de la disponibilidad de servicios en la productividad por trabajador. Para ello será implementado un modelo con la siguiente estructura:

$$
\Delta P M G l_{i, t}=\alpha+X_{i, t} \beta+\gamma_{i, t}
$$

donde $\triangle P M G l_{i, t}$ indica el crecimiento en la producción por trabajador al año del país $i$ y el año $t ; X_{i, t}$; es un conjunto de variables que afectan la productividad, tales como el crecimiento del valor agregado de las actividades económicas de los sectores primario, secundario y terciario, así como el crecimiento de las importaciones de bienes y servicios. Nótese que adicional a la actividad de servicios se ha incluido la de otros sectores, como el primario y el secundario; dicha formulación busca evitar la omisión de los diferentes insumos de la producción de los tres sectores económicos que pueden tener diferentes intensidades en países con estructuras productivas particulares. Finalmente, en $\gamma_{i, t}$ se encuentran los efectos fijos o aleatorios del modelo de panel.

Para dicho modelo se ha construido una base de datos con información de 124 países y un periodo de 36 años (1971-2007). Las series utilizadas corresponden, fundamentalmente, a información de cuentas nacionales, comercio de bienes y servicios, y al valor agregado de los diferentes sectores productivos de los países. La información recopilada proviene de la División de Estadísticas de las Naciones Unidas y el Banco Mundial. Las series tienen como unidad de medida los dólares corrientes y el análisis se ha elaborado en términos de crecimientos (porcentajes).

Como se puede observar en la tabla 1, las variables relacionadas con el valor agregado de los diferentes sectores de la economía utilizan como convención la clasificación ISIC versión 3.1. De este conjunto de cuentas se ha seleccionado solo un conjunto de variables representativas de la producción primaria (A-B), de la producción manufacturera (D) y de la producción de servicios considerados como de alta tecnología y capital humano (J, K, L, M, N, O, P) (Niño, 2005; Li, Greenaway \& Hine, 2005). Además, la productividad por trabajador del país es representada como el valor agregado total generado por trabajador y por año. 
Desarrollo del sector servicios y su papel en la consolidación del crecimiento económico mundial

ANDRÉS FERNANDO JOLA SÁNCHEZ

Tabla 1.

Clasificación ISIC 3.1

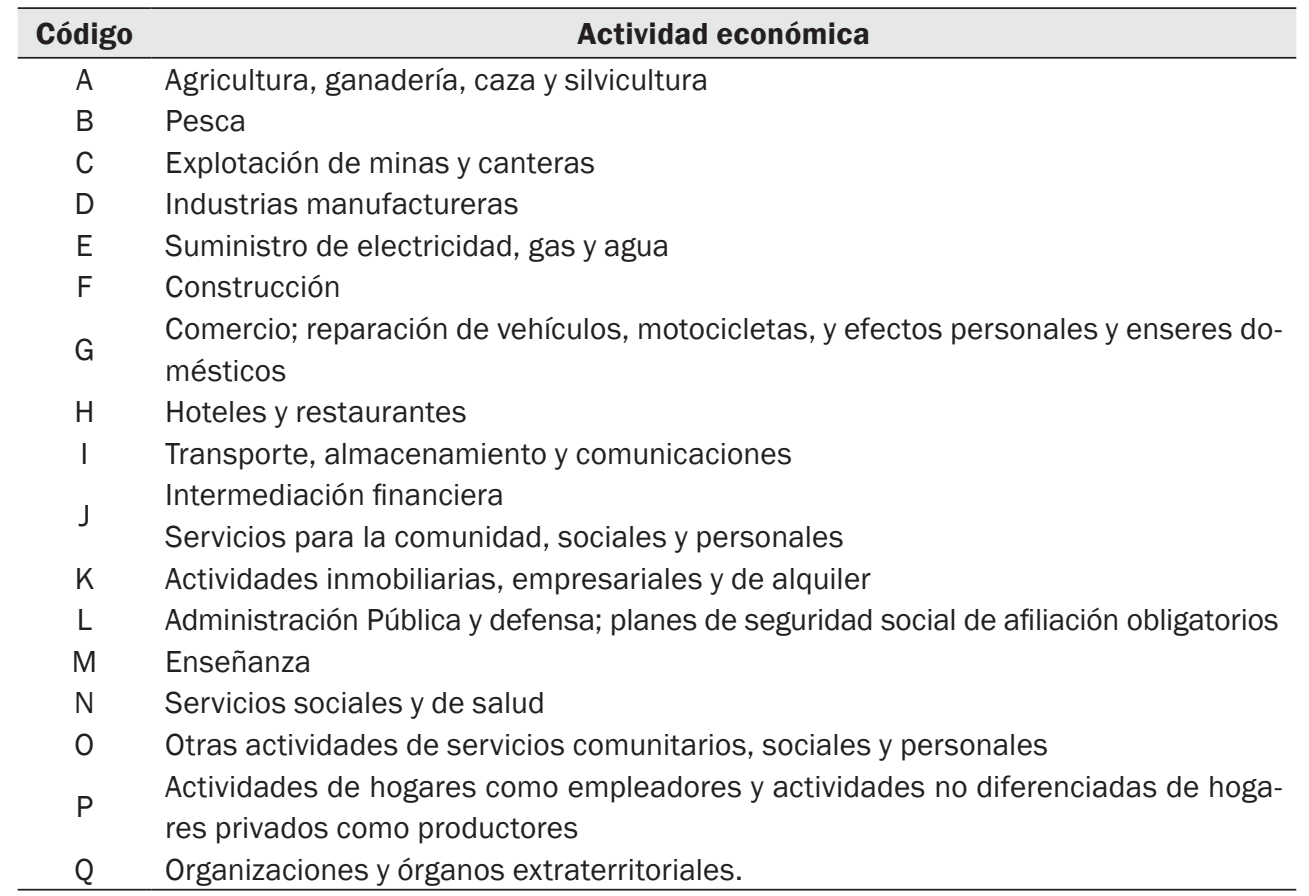

Fuente: División de Estadísticas de las Naciones Unidas

Para el comercio de bienes y servicios fue utilizado el registro agregado de exportaciones e importaciones del Banco Mundial, cuya metodología se basa en la de balanza de pagos. En el caso de servicios, la actividad comercial se centra en servicios de transporte, viajes, comunicaciones, seguros, financieros, derechos de licencias, personales, culturales y recreacionales, así como servicios del Gobierno y otros servicios empresariales.

En la tabla 2 se presentan algunas estadísticas descriptivas del conjunto de variables utilizadas. En total, se tomaron 4.588 observaciones que fueron el resultado de una depuración de los datos por país, en la que como regla se eliminaron países con datos incoherentes y anómalos, y todos aquellos donde existiera más de un 50\% de información faltante. Por esta vía se eliminaron cerca de 64 países. Adicionalmente, algunos datos faltantes de la variable dependiente (productividad por trabajador) y de las variables asociadas con la producción de bienes y servicios fueron completados, con el uso 
de interpolación lineal, en el caso de las variables dependientes, ${ }^{2}$ y con la imputación de datos por variable ${ }^{3}$ en el caso de la variable dependiente. Se estima que el número de datos agregados por esta vía fue máximo el $2 \%$ del total de datos de este estudio.

Tabla 2.

Estadísticas descriptivas de las variables de interés (valores corrientes expresados en logaritmos)

\begin{tabular}{lcccccc}
\hline \multicolumn{7}{c}{ Muestra: 1971-2007 } \\
\hline Media & PMT & Agric. & Manuf. & Serv. & Imp. B & Imp. S \\
Mediana & 0.0546 & 0.0581 & 0.0791 & 0.0839 & 0.0774 & 0.0805 \\
Máximo & 1.8295 & 0.9383 & 1.6257 & 1.1224 & 1.9264 & 2.8824 \\
Mínimo & -1.7232 & -1.6383 & -1.8182 & -1.1575 & -1.5996 & -1.3898 \\
Desv. Estd & 0.1415 & 0.1644 & 0.1769 & 0.1524 & 0.1793 & 0.1912 \\
Skewness & -0.7619 & -0.8075 & -0.3970 & -0.9893 & -0.4672 & 0.7772 \\
Kurtosis & 19.896 & 9.5816 & 13.625 & 10.963 & 11.615 & 20.800 \\
Jaque-Bera & 55.021 & 8.779 .6 & 21.703 & 12.871 & 12.044 & 51.217 \\
P-value & 0.0000 & 0.0000 & 0.0000 & 0.0000 & 0.0000 & 0.0000 \\
Sum & 250.75 & 266.82 & 363.20 & 385.13 & 298.02 & 310.05 \\
Sum Var. & 91.851 & 124.09 & 143.62 & 106.66 & 123.73 & 140.76 \\
Obs. & 4.588 & 4.588 & 4.588 & 4.588 & 3.849 & 3.850 \\
Faltantes & 0 & 0 & 0 & 0 & 739 & 738 \\
\hline
\end{tabular}

Fuente: UNSTAT, Banco Mundial, elaboración propia.

Por último, los países fueron clasificados según su nivel de ingresos, de acuerdo con los criterios del año 2007 del Banco Mundial.

El modelo de panel, como se ha mencionado, será la técnica para analizar el conjunto de datos por país, periodos y niveles de ingreso. Dicha técnica permitirá evitar los problemas de estimación econométrica relacionados con variables omitidas invariantes en el tiempo (Mayorga y Muñoz, 2000; Sosa, 2004), así como lograr discriminar tanto los

2 Se completaron datos faltantes al interior de la serie de tiempo, es decir, no se completaron datos al inicio o final de la serie.

3 La variable proxy para la imputación de la productividad por trabajador fue la productividad per cápita. 
Desarrollo del sector servicios y su papel en la consolidación del crecimiento económico mundial

ANDRÉS FERNANDO JOLA SÁNCHEZ

efectos fijos como aleatorios entre las unidades transversales y longitudinales. Baltagi (2008) resalta otras ventajas de la técnica, como el mejoramiento de la eficiencia de la estimación, el reconocimiento de que las unidades transversales son heterogéneas y, además, el análisis de series temporales, incorporando con ello las dinámicas de transición y los efectos intertemporales.

La estimación de un modelo tipo panel incluye efectos fijos o aleatorios en unidades transversales o longitudinales de la siguiente forma:

$$
Y_{i, t}=X_{i, t} \beta_{i, t}+\gamma_{i, t}
$$

donde representa una unidad transversal, indica la dimensión longitudinal, y ambas tienen valores que van desde 1 hasta $\mathrm{N}$ y $\mathrm{T}$, respectivamente.

$X_{i, t}$ es el conjunto de variables transversales explicativas del modelo, que son relacionadas mediante $\mathrm{K}$ parámetros de regresión $\beta_{i, t}$, tanto por unidad transversal como longitudinal. Finalmente, el error del modelo $\gamma_{i, t}(11)$ se denota como el conjunto de efectos fijos o aleatorios en la dimensión temporal o transversal, donde $\eta_{i}$ representa los efectos fijos o aleatorios por unidad transversal, al tiempo que $\tau_{t}$ son los efectos fijos o aleatorios en la estructura temporal. Finalmente $e_{i, t}$ representa el error que supone ser normal, independiente e idénticamente distribuido.

$$
\gamma_{i, t}=\eta_{i}+\tau_{t}+e_{i, t}
$$

Bajo el modelo de efectos fijos, la estimación al interior de las secciones transversales (estimador within) con un único coeficiente se puede describir así:

$$
\hat{\beta}=\left(\sum_{i=1}^{N} \sum_{t=1}^{T} x_{l, t}^{i} x_{i, t}\right)^{-1}\left(\sum_{i=1}^{N} \sum_{t=1}^{T} \ddot{x}_{l, t}^{i} \ddot{y}_{i, t}\right)
$$

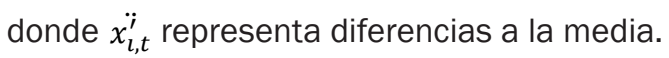

En la estimación econométrica utilizando diferencias a la media (12), el estimador de los coeficientes de regresión serán insesgados condicionalmente a $X$ y eficientes. Sin embargo, $\hat{\beta}$ y $\eta_{i}$ y solo serán consistentes si para un T fijo, $N \rightarrow \infty$, o, si $T \rightarrow \infty$ para cada una de las secciones transversales. 
Varios autores, como Beck y Katz (1995), han propuesto algunas metodologías de estimación para hacer frente a la violación de algunos de los supuestos centrales del modelo tradicional.

Uno de los más reconocidos, y de hecho implementado en este documento, es el modelo de errores estándares de panel corregidos (PCSE). Esta metodología permite incorporar las mejores estimaciones sobre la matriz de varianza de los estimadores de $\beta$ (Johnson, 2004). El modelo lleva a cabo una transformación de la matriz de varianza de los estimadores de $\beta$ con información que tiene en cuenta tanto la posible autocorrelación entre las diferentes unidades y periodos, como la distinta varianza de las unidades de la matriz de varianza-covarianza de los residuales.

En notación, la matriz de varianza-covarianza de los residuales $(\Omega)$ en un modelo balanceado puede representarse de la siguiente forma, teniendo en cuenta tanto la correlación contemporánea como la heterocedasticidad:

$E\left(\epsilon \epsilon^{\prime}\right)=\Omega=\sum \mathrm{m} \times \mathrm{m} \otimes \mathbf{I}_{\mathrm{T} \times \mathrm{T}}$

donde $\mathbf{I}_{\mathrm{ij}}$ es igual a un matriz identidad

Aunque la estimación requerirá que se transforme la matriz de varianza de los estimadores de los coeficientes del modelo, los coeficientes no cambiarán dado que no están sesgados (asumiendo que el valor esperado de los errores es cero). No obstante, el modelo PCSE reestima $E\left(\epsilon \epsilon^{\prime}\right)$ permitiendo correlaciones contemporáneas y heterocedasticidad. En particular, generando un $\widehat{\Omega}$ de la siguiente forma:

$$
\widehat{\Omega}=\frac{\epsilon \epsilon^{\prime}}{\mathrm{T}} \otimes \mathbf{I}_{\mathrm{T}}
$$

donde la estimación de la varianza de los coeficientes se describirá así:

$$
\operatorname{Var}(b)=\left(x^{\prime} x\right)^{-1} \widehat{\Omega} x^{\prime} x\left(x^{\prime} x\right)^{-1}
$$

En caso de que exista autocorrelación en los residuales deberá reestimarse previamente del modelo debido a que la metodología de PCSE asume que estos errores no se encuentran autocorrelacionados, por lo tanto, en este escenario se deberá implementar un método para aplicar PCSE como el de Prais-Winsten (Johnston, 1989). 
Desarrollo del sector servicios y su papel en la consolidación del crecimiento económico mundial

ANDRÉS FERNANDO JOLA SÁNCHEZ

En resumen, la técnica a utilizar en este trabajo será PCSE, ajustado por los efectos adecuados en los componentes de error, y corrigiendo por los problemas que se presentasen al momento de la estimación según las descripciones metodológicas anotadas.

\section{Resultados}

\subsection{Desarrollo del sector servicios y crecimiento económico en el mundo}

Un elemento central en este estudio es relacionar la productividad por trabajador y el crecimiento económico. De acuerdo con la teoría planteada, existe una asociación positiva entre estas dos variables. Así, y como un primer resultado de este documento, se encuentra en la base de datos conformada una alta correlación entre el crecimiento del producto por trabajador y el crecimiento económico para todos los niveles de ingreso, tal y como se muestra en el grupo de figuras 2 . Un análisis de correlaciones cruzadas indica que la correlación de estas dos variables es 0,93, es decir, una relación prácticamente uno a uno entre las variables.

\section{Figura 2.}

Crecimiento económico y productividad por trabajador según diferentes niveles de ingreso

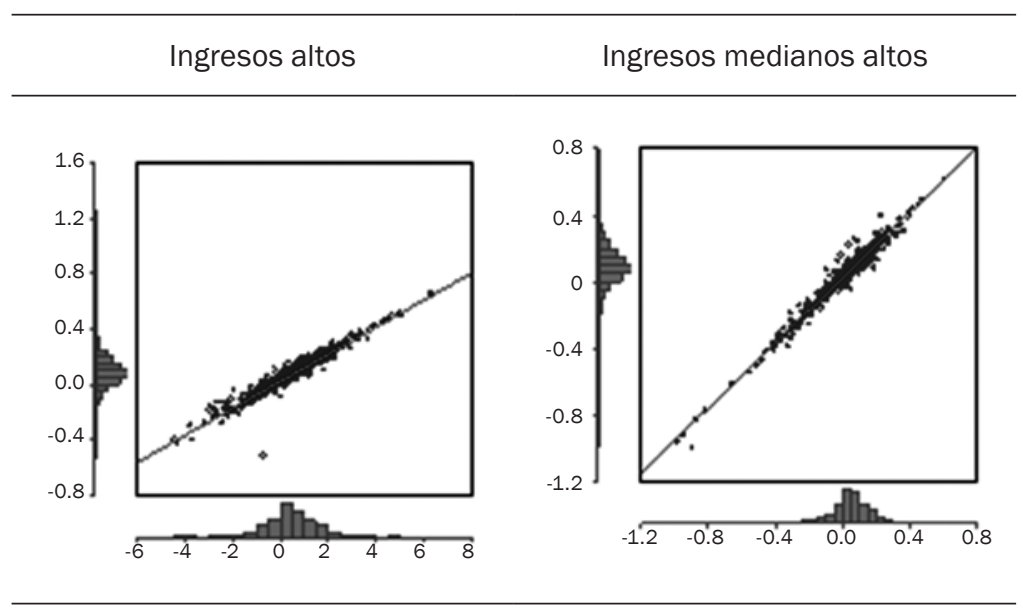



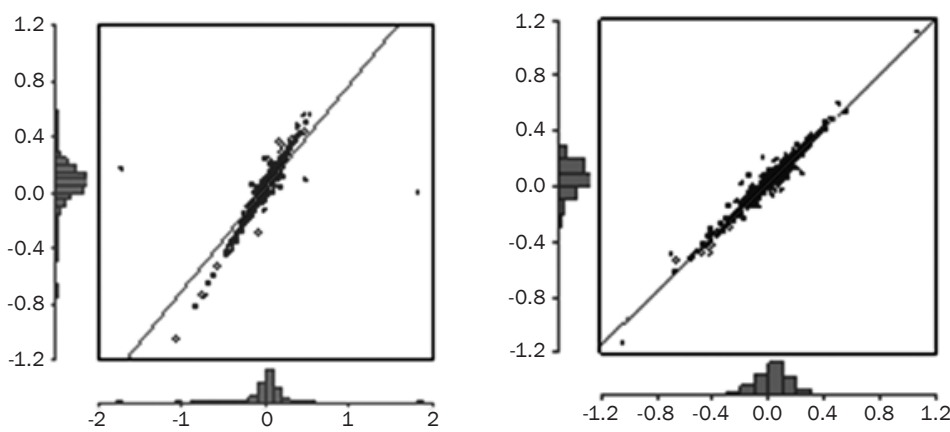

Nota: se muestra el crecimiento económico en las ordenadas, y crecimiento de la productividad por trabajador en las abscisas.

Fuente: elaboración propia

Dado que el producto por trabajador está relacionado directamente con el crecimiento económico, el objetivo se centrará en mostrar que la productividad por trabajador se ve afectada positivamente por la importación y producción interna de servicios.

El modelo tipo panel que se utilizó en este estudio corresponde a uno de efectos fijos. En línea con lo establecido en la sección anterior, no solo la naturaleza de los datos seleccionados, sino también la corroboración estadística utilizando el test de Hausman, validan un modelo de este tipo (al rechazarse la hipótesis nula de que la diferencia entre los modelos de efectos fijos y aleatorios no es sistemática; ver tabla 3).

Tabla 3.

Efectos aleatorios correlacionados - Test de Hausman

\begin{tabular}{lccc}
\hline \multicolumn{1}{c}{ Resumen del test } & Chi-Sq. Estad. & Chi-Sq. d.f. & Prob. \\
\hline $\begin{array}{l}\text { Secciones transversales-efectos } \\
\text { aleatorios }\end{array}$ & 50.609759 & 5 & 0.0000 \\
\hline
\end{tabular}

Fuente: elaboración propia

Otros tests sobre las unidades transversales y longitudinales sugieren que un modelo de efectos fijos en los países y en los periodos es la opción adecuada para el modelo 
Desarrollo del sector servicios y su papel en la consolidación del crecimiento económico mundial

ANDRÉS FERNANDO JOLA SÁNCHEZ

planteado, dado que se rechaza la hipótesis nula de que los efectos en las unidades y los periodos sea significativamente igual a cero, tal como lo muestran los estadísticos Chi-cuadrado y $\mathrm{F}$ de las últimas dos filas de la tabla 4.

Tabla 4.

Test de efectos fijos en secciones transversales y periodos

\begin{tabular}{llll}
\hline \multicolumn{1}{c}{ Test de efectos } & \multicolumn{1}{c}{ Estadístico } & \multicolumn{1}{c}{ GL } & \multicolumn{1}{c}{ Prob. } \\
\hline Sección transversal F & 1.480 & $(123,3684)$ & 0.0006 \\
Sección transversal Chi-cuadrado & 185.741 & 123 & 0.0002 \\
Periodos F & 1.488 & $(36,33684)$ & 0.0308 \\
Periodos Chi-cuadrado & 55.571 & 36 & 0.0196 \\
Sección transversal / Periodo F & 1.502 & $(159,3684)$ & 0.0000 \\
Sección transversal /Periodo Chi-square & 241.817 & 159 & 0.0000 \\
\hline
\end{tabular}

Fuente: elaboración propia

Un modelo correctamente especificado hace necesaria la demostración de que se cumplen todos los supuestos sobre los residuales y los datos del modelo. Al efectuar las pruebas que verifican los diferentes tipos de problemas de estimación, se concluye que el modelo two way tiene problemas de heterocedasticidad y autocorrelación de los residuales de orden uno. Por lo anterior, y de acuerdo con la metodología de la sección 4, se implementó el modelo con la corrección de errores de panel-PCSE. No obstante, al llevar a cabo las estimaciones con los diferentes tipos de modelo posibles: efectos fijos (a), efectos aleatorios (b) y two way PCSE (c), los coeficientes, como se señala en las tablas 5 y 6 , no demuestran en ningún caso cambios drásticos en la significancia, ni en el signo o la magnitud.

Los resultados del total de países (tabla 5) indican que existe evidencia significativa para concluir que la disponibilidad de servicios es un canal de crecimiento de la productividad por trabajador. Existen dos mecanismos para la transmisión de este efecto, vía actividad comercial, por un lado y, por el otro, vía producción nacional de bienes y servicios. En ambos casos se encuentra que la actividad de servicios es un determinante del crecimiento de la productividad por trabajador. 


\section{Tabla 5.}

Estimaciones usando efectos fijos y aleatorios

\begin{tabular}{ccc}
\hline & $\begin{array}{c}\text { Efectos fijos } \\
\text { (a) }\end{array}$ & $\begin{array}{c}\text { Efectos aleatorios } \\
(\mathrm{b})\end{array}$ \\
\hline Constante & $-0,02063$ & $-0,01415$ \\
$\Delta$ Imp. bienes & $0,03064 * * *$ & $0,02998 * * *$ \\
$\Delta$ Imp. serv. & $0,01058 *$ & $0,01380 * *$ \\
$\Delta$ VA. agric. & $0,18395 * * *$ & $0,17690 * * *$ \\
$\Delta$ VA. manuf. & $0,31870 * * *$ & $0,31589 * * *$ \\
$\Delta$ VA serv. AT & $0,39251 * * *$ & $0,39928 * * *$ \\
R-cuadrado global & 0,8184 & 0,8159 \\
F-Chi2 & $415,22 * * *$ & $17071,9 * * *$ \\
Observaciones & 3.849 & 3.849 \\
Países incluidos & 124 & 124 \\
\hline
\end{tabular}

Significancia al $* * * 1 \%, * * 5 \%, * 10 \%$

Fuente: elaboración propia

En el conjunto de variables comerciales (importación de bienes y servicios) se estima que, todo lo demás constante, un 1,725\% de un incremento de un 1\% de la importación de servicios se transmite a un crecimiento de la productividad. Es decir, si la importación de servicios se incrementa un 10\% entre un periodo y otro, la productividad por trabajador se incrementará un 0,1725\% en este mismo periodo. Para la importación de bienes, dado el mismo análisis, este valor corresponde a 2,494\%.

Las variables de la producción nacional indican que, para servicios catalogados como de alto conocimiento (VA Servicios AT), cerca de un 40\% de un 1\% de crecimiento de la actividad del sector es transmitida al crecimiento de la productividad por trabajador, cifra que supera ampliamente los sectores de manufactura y agricultura, con valores de $31,2 \%$ y $17,8 \%$ respectivamente. 
Desarrollo del sector servicios y su papel en la consolidación del crecimiento económico mundial

ANDRÉS FERNANDO JOLA SÁNCHEZ

Tabla 6.

Estimaciones econométricas para todos los países

\begin{tabular}{cc}
\hline \multicolumn{2}{c}{ Efectos fijos PCSE (c) } \\
Muestra completa (1) \\
\hline Constante & $-0,01590 * * *$ \\
$\Delta$ Imp. bienes & $0,02494 * * *$ \\
$\Delta$ Imp. servicios & $0,01725 * *$ \\
$\Delta$ VA. agricultura & $0,17798 * * *$ \\
$\Delta$ VA. manufactura & $0,31858 * * *$ \\
$\Delta$ VA servicios AT & $0,39482 * * *$ \\
R-cuadrado global & 0,8413 \\
F-Chi2 & $19684,05 * * *$ \\
Observaciones & 3.849 \\
Países incluidos & 124 \\
\hline
\end{tabular}

Significancia al $* * * 1 \%, * * 5 \%, * 10 \%$

Fuente: elaboración propia

\subsection{Desarrollo del sector servicios y crecimiento económico en países con diferentes niveles de ingreso}

Los resultados de esta sección se mostrarán bajo la clasificación de ingresos del Banco Mundial, a saber: ingresos altos, medianos altos, medianos bajos, y bajos. Además, distinguiendo entre resultados asociados al comercio internacional y producción nacional.

Para el grupo de variables relacionadas con el comercio internacional, las salidas del modelo indican que en los países de ingresos altos la importación de bienes y servicios representa uno de los mayores aportes a la productividad en los diferentes grupos de países. Se estima que un 1,46\% del crecimiento de bienes y un 3,42\% del de servicios son incorporados al crecimiento de la productividad por trabajador. Es decir, la importación de servicios, además de ser un canal de crecimiento de la productividad, en este grupo de países su valor más que duplica el aporte de la importación de bienes. Dicha observación es cierta también en los países de bajos ingresos, en los cuales, el crecimiento de la importación de servicios transmite cerca de un 30\% adicional al producto por trabajador sobre el efecto que hace la importación de bienes. En el caso de países de ingresos medianos altos y medianos bajos, aunque se observa el efecto opuesto, la 
significancia de los coeficientes relacionados con la importación de servicios hace débil la interpretación de estos valores.

Por otro lado, el conjunto de variables relacionadas con la producción nacional de bienes y servicios demuestra algunos hechos interesantes. En primer lugar, se observa que el aporte de los diferentes sectores al crecimiento de la productividad es diferente, según el nivel de ingresos. En los países de ingresos altos cerca de un 54,55\% del crecimiento en los servicios es incorporado al crecimiento de la producción por trabajador. Es decir, todo lo demás constante, un 1\% de crecimiento en la producción de sectores de servicios de alta tecnología, generará cerca de un 0,5455\% de crecimiento directo en la productividad por trabajador. No obstante, mientras el aporte de la manufactura se mantiene relativamente alto, en $35,81 \%$, en el sector de agricultura el valor es de hecho negativo.

Tabla 7.

Estimaciones utilizando PCSE. Actividad de servicios

y productividad para ingresos altos y medianos altos.

\begin{tabular}{lll}
\hline \multicolumn{1}{c}{ Ingresos } & \multicolumn{1}{c}{ Altos (2) } & \multicolumn{1}{c}{ Medianos altos (3) } \\
\hline Constante & $-0,01909 * * *$ & $-0,03299 * *$ \\
$\Delta$ Imp. bienes & 0,01459 & $0,03981 * * *$ \\
$\Delta$ Imp. servicios & $0,03418 * *$ & 0,00821 \\
$\Delta$ VA. agricultura & $-0,03364 * *$ & $0,13927 * * *$ \\
$\Delta$ VA. manufactura & $0,35814 * * *$ & $0,3862 * * *$ \\
$\Delta$ VA servicios AT & $0,54555 * * *$ & $0,42905 * * *$ \\
R-cuadrado global & 0,9086 & 0,9589 \\
Covarianzas & 666 & 406 \\
Correlaciones & 36 & 28 \\
Wald-Chi2 & $5438,92 * * *$ & $65528,40 * * *$ \\
Observaciones & 1.201 & 860 \\
Países incluidos & 36 & 28 \\
\hline
\end{tabular}

Significancia al *** $1 \%, * * 5 \%, * 10 \%$

Fuente: elaboración propia

Para los países de ingresos medianos altos el resultado del estudio muestra que en los servicios catalogados de alta tecnología, un 40\% del crecimiento en estos servicios se 
Desarrollo del sector servicios y su papel en la consolidación del crecimiento económico mundial

ANDRÉS FERNANDO JOLA SÁNCHEZ

traduce en crecimiento en la productividad; en los sectores de manufactura y agricultura Ios valores son de hecho mayores, dichas estimaciones se acercan al 38,62\% y 13,93\% respectivamente. Por lo tanto, se observa que en los países de ingresos medianos altos el sector manufacturero tiene el mayor impacto en la productividad por trabajador que en cualquier otro conjunto de países; sin embargo, el aporte de los servicios al interior de este grupo de países es todavía el más importante.

En los países con ingresos medianos bajos la dependencia en la agricultura es más fuerte, y además, el crecimiento de la productividad por cuenta de un aumento en los servicios y manufactura es menor que en los casos anteriores. No obstante, en estos países el aporte de los servicios es superior al que hace la manufactura.

\section{Tabla 8.}

Estimaciones utilizando PCSE. Actividad de servicios y productividad para ingresos medianos bajos y bajos.

\begin{tabular}{lll}
\hline \multicolumn{1}{c}{ Ingresos } & \multicolumn{1}{c}{ Medianos bajos (4) } & \multicolumn{1}{c}{ Bajos (5) } \\
\hline Constante & $-0,03019$ & $-0,02582$ \\
$\Delta$ Imp. bienes & $0,03932 * * *$ & $0,01161 *$ \\
$\Delta$ Imp. servicios & 0,00316 & $0,01512 * *$ \\
$\Delta$ VA. agricultura & $0,22415 * * *$ & $0,42743 * * *$ \\
$\Delta$ VA. manufactura & $0,32418 * * *$ & $0,18275 * * *$ \\
$\Delta$ VA servicios AT & $0,34729 * * *$ & $0,27525 * * *$ \\
R-cuadrado global & 0,7404 & 0,9321 \\
Covarianzas & 666 & 300 \\
Correlaciones & 36 & 24 \\
Wald-Chi2 & $4374,80 * * *$ & $124651 * * *$ \\
Observaciones & 1.086 & 702 \\
Países incluidos & 36 & 24 \\
\hline
\end{tabular}

Significancia al $* * * 1 \%, * * 5 \%, * 10 \%$

Fuente: elaboración propia

Por último, para el grupo de países de ingresos bajos se demuestra que un $42,73 \%$ del crecimiento en la agricultura es incorporado en el crecimiento de la productividad por trabajador. Dicho valor es superior al hallado en cualquier otro grupo de países, observando así la más alta dependencia en el sector primario. No obstante, dado que en 
estos países probablemente no existen las estructuras en la producción nacional para generar servicios con alto contenido en capital humano, se observa que el crecimiento en la importación de servicios es, en cambio, un mecanismo que aporta al producto por trabajador un valor que supera al de todos los grupos de países, a excepción de los de ingresos altos. Esto podría implicar que en los países pobres la importación de servicios incorpora los conocimientos y el capital humano que no es posible incorporar por medio de la producción interna.

\section{Conclusiones}

En este documento se ha examinado la relación entre el sector de servicios, el producto medio por trabajador y el crecimiento económico. La hipótesis planteada sugiere que los servicios, siendo un medio para el crecimiento de la frontera tecnológica, crean las condiciones para hacer más productiva las actividades de la economía. En la estructura conceptual planteada, un mayor nivel de servicios intensivos en capital humano y tecnología permite que la economía sea más competitiva y que, de esta forma, tenga una acelerada senda de crecimiento económico. Por esta razón, tanto la importación de servicios como la producción nacional de ellos son dos canales a través de los cuales es posible incrementar la oferta de servicios, disponibles para firmas, hogares y consumidores internacionales.

Con el propósito de hallar evidencia de lo anterior, se llevó a cabo una estimación de tipo panel en la cual, estimando efectos fijos en los países y en los periodos, se determinó la relación entre la actividad de servicios y el producto por trabajador. Dicho panel contiene información para más de 124 países y periodos que van de 1970 a 2007. La estimación requirió el uso de metodologías econométricas ajustadas a los problemas tanto de heterocedasticidad, como de autocorrelación y correlación contemporánea.

Uno de los principales hallazgos de la estimación econométrica fue haber determinado que los servicios intensivos en capital humano y tecnología aportan un valor significativo y positivo al crecimiento de la productividad por trabajador en el grupo total de países estudiados. Adicionalmente, que la apertura comercial, vista en las importaciones tanto de bienes como de servicios, son mecanismos confiables estadísticamente que aceleran el crecimiento de la productividad por trabajador. 
Desarrollo del sector servicios y su papel en la consolidación del crecimiento económico mundial

ANDRÉS FERNANDO JOLA SÁNCHEZ

Se encontrado también que, dados los diferentes niveles de ingresos, el crecimiento de la productividad por incrementos en la disponibilidad de servicios es mayor cuanto mayor sean los ingresos de los países. El crecimiento de la productividad de los países de menores ingresos se halla fundamentado en el crecimiento del sector primario, y los países cuyos ingresos son medianos altos tienen la mayor relación positiva en la producción de manufacturas como fuente del crecimiento de la productividad por trabajador.

En términos generales, este hallazgo indica que un tema de primera importancia en la consolidación de políticas públicas será no solo el fomento de la industria nacional de servicios de alta tecnología, sino también la puesta en marcha de estrategias con la finalidad de que muchos de estos servicios estén disponibles para la economía doméstica y puedan ser incorporados a la cadena de valor de la economía nacional mediante el proceso de importación. De acuerdo con la evidencia de este documento, el desmonte de barreras al comercio internacional de servicios y la gestión de una efectiva política de desarrollo de este sector traerán ventajas directas a la productividad, la competitividad y al deseado crecimiento económico alto y sostenido.

\section{Referencias}

BaltaGl, B. (2008). Econometric analysis of panel data. Nueva York, NY, EE. UU.: John Wiley \& Sons.

BAnga, R. \& Goldar, B. (2004). Contribution of services to output growth and productivity in India manufacturing: pre and post reforms. Indian Council for Research on International Economic Relations, 33.

Bhagwati, J. (1984, junio). Splintering and Disembodiment of Services and Developing Nations. The World Economy.

Box, G.; Jenkins, G. \& Reinsel, G. (1994). Time Series Analysis: forecasting and control. Nueva Jersey, NJ, EE. UU.: Prentice-Hall, Englewood Cliffs.

Demirguc-Kunt, A. \& Levine, R. (1999). Bank-based and market-based financial systems cross-country comparisons. Policy Research Working Paper Series 2143, The World Bank. 
DuRLAUf, S. (2001). Manifiesto for growth econometrics. Journal of Econometrics. Elsevier.

Eschendach, F. \& Hoekman, B. (2005). Services Policy Reform and Economic Growth in Transition Economies, 1990-2004. París, Francia: Groupe d'e Economie Mondiale, Institut d'e Politiques, World Bank y CPR, 20.

Fernandes, A. \& Paunov, C. (2008). Services FDI and Manufacturing Productivity Growth: There is a link. The World Bank Development Research Group, University of London Economics, 56.

García, C. E. \& Menéndez, L. S. (1992). Conceptos y clasificaciones en la economía de Ios servicios. Documento de Trabajo 92-07. Madrid: España, Instituto de Estudios Sociales Avanzados CSIC.

Hoekman, B. \& Mattoo, A. (2000, marzo). Services, economic development and the next round of negotiations in services. Journal of International Development, 12(2), 283-296.

JUDSON, R. \& OwEn, A (1999). Estimating dynamic panel data models: a guide for macroeconomists. Federal Reserve of Governors, Economics Letters-Elsevier

Johnson, P. (2004). Cross Sectional Time Series: The Normal Model and Panel Corrected Standards Errors.

Khoury, A. \& Savvides, A. (2006). Openess in Services Trades and Economic Growth, 10.

KIVIET, J. (1995). On bias, inconsistency, and efficiency of various estimators in dynamic panel data models. Journal of Econometrics. Elsevier.

Kravis, I.; Heston, A. \& Summers, R. (s. f.). The Share of Services in Economic Growth, 32.

Kristensen, I.O. \& WaWro, G. (2003). Lagging the Dog?: The Robusteness of Panel Corrected Standard Errors in the Presence of Serial Correlation and Observation Specific Effects. Columbia University, Department of Political Science.

LI, X.; Greenaway, D. \& Hine, R. (2005). Importaciones de servicios y crecimiento económico. Un análisis dinámico de panel. Competitividad e internacionalización de los servicios en los países europeos (824), 18. 
Desarrollo del sector servicios y su papel en la consolidación

del crecimiento económico mundial

ANDRÉS FERNANDO JOLA SÁNCHEZ

Mauleon, I. (1987). Problemas prácticos en el tratamiento econométrico de datos cross sectios. Investigaciones Económicas (segunda época), (XI), 1, 41-94.

MAYoRga \& Muñoz (2000). La técnica de datos de panel. Una guía para su uso e interpretación. Costa Rica: Banco Central, Departamento de Investigaciones Económicas.

Neira, I.; VieiRa, E. \& Lamelas, N. (s. f.). Educación, investigación, desarrollo económico y social: análisis regional europeo, con especial referencia a España y Portugal. Santiago de Compostela, España: Universidad de Santiago de Compostela, Facultad de Econometría Aplicada.

NicolettI \& SCARPETTA (2003). Regulation, productivity and growth: OECD evidence. Economic Policy, CEPR \& CES \& MSH, (18), 36, 9-72.

Niño, J. A. (2005). Sector servicios y desarrollo económico. Bogotá, Colombia: Universidad Nacional de Colombia.

OECD (2005). Growth in Services fostering employment, productivity and innovation. OECD publications, 23.

PeÑA, D. (2005). Análisis de series temporales. Madrid, España: Alianza.

Stata Longitudinal/Panel Data Reference Manual Release 10 (2007). Stata press publication. Texas, T., EE. UU.: College Station Texas.

Sosa E.W. (2004, 19 de mayo). Econometría de datos en paneles. (Presentación). Córdoba: Argentina: Universidad Nacional de Córdoba.

WAVerman \& Roller (2001, septiembre). Telecommunications Infrastructure and Economic Development: A Simultaneous Approach. American Economic Review, 91(4), 909-923.

Williamson, S.D. (2008). Macroeconomics. Pearson Addison Wesley.

WooldRIDGE, J. (2002). Econometric Analysis of Cross Section and Panel Data. MIT Press, 247-291. 